\title{
Medicalização e migrações internacionais: proteção de direitos e práticas de saúde no controle de fronteiras
}

Medicalization and international migration: protection of rights and health practices at border control

Medicalización y migración internacional: protección de derechos y prácticas de salud en el control de fronteras

Moisés Moreira Vieira ${ }^{1}$

RESUMO: O trabalho tem como objetivo analisar os impactos da medicalização no controle de fronteiras dos países, identificando possíveis implicações para a prática médica e para os direitos dos migrantes internacionais. Para tanto, assume como objeto de análise as restrições de viagem relacionadas ao HIV/AIDS, existentes na legislação imigratória do Canadá. No que tange à metodologia utilizada, o estudo classifica-se como bibliográfico e documental. Quanto aos procedimentos metodológicos empregados, têm-se a leitura e a análise de materiais publicados sobre o tema, tais como livros, publicações em periódicos e artigos científicos, além do estudo e interpretação de informações que se encontram em documentos sem conteúdo analítico (leis, relatórios com dados estatísticos, etc.), conferindo-Ihes importância como fonte de análise. O estudo verifica que a medicalização do controle de fronteiras obscurece a aferição da eficácia das restrições, enquanto instrumento de proteção da população, capaz de justificar o sacrifício de direitos dos migrantes soropositivos. Além disso, observa-se que o aumento da jurisdição dos profissionais da saúde contribui para o esvaziamento terapêutico da atividade médica, centrada em rotinas administrativas em detrimento do melhor interesse do migrante/paciente. Finalmente, nota-se que discursos e práticas médicas conhecem e produzem o migrante "saudável", apto a cruzar as fronteiras do Estado, num contexto de relações de poder, que violam direitos dos migrantes soropositivos.

Palavras-chave: Medicalização. Migração. Saúde. Direitos humanos.

ABSTRACT: This paper aims to analyze the impacts of medicalization at border control, identifying possible implications for medical practice and international migrants' rights. For this purpose, it studies HIV-related restrictions imposed by Canada's immigration law. As for the methodology, the research is bibliographical and documental. Concerning methodological procedures, the paper draws on the reading and analysis of published materials on the subject, such as books, academic articles and papers, along with the interpretation of information found in documents without analytical content (laws, statistical reports, etc), granting them importance as a source of analysis. The paper demonstrates that medicalization of border control obscures the determination of the efficacy of such restrictions as a means to protect the population, capable of justifying the sacrifice of HIVpositive migrants. Furthermore, it argues that the expansion of health professionals' jurisdiction contributes to the therapeutical emptying of medical practices, centered around

\footnotetext{
${ }^{1}$ Mestre em Relações Internacionais pela Universidade Federal da Bahia (UFBA). Ex-bolsista da Fundação de Amparo à Pesquisa da Bahia (FAPESB). Graduado em Direito pela UFBA. Advogado. Salvador/Bahia, Brasil. E-mail:m.moreira.vieira@gmail.com
} 
administrative routines to the detriment of the best interest of migrants/patients. Finally, the paper contends that medical discourses and practices enable the knowledge and production of the "healthy" migrant, able to cross states' borders, which violate HIV-positive migrants' rights.

Keywords: Medicalization. Migration. Health. Human rights.

RESUMEN: El estudio tiene como objetivo analizar los impactos de la medicalización en el control de las fronteras de los países, identificando las posibles implicaciones para la práctica médica y para los derechos de los migrantes internacionales. Para este propósito, se toma como objeto de análisis las restricciones de viaje relacionadas con el VIH / SIDA, presentada por la legislación de inmigración existente en Canadá. En cuanto a la metodología utilizada, el estudio se clasifica como bibliográfico y documental. Acerca de los procedimientos metodológicos empleados, han sido la lectura y el análisis de los materiales publicados sobre el tema, tales como libros, publicaciones en revistas y artículos científicos, además del estudio y la interpretación de las informaciones que se encuentran en los documentos sin contenido analítico (leyes, informes con datos estadísticos, etc.), dándoles importancia como fuente de análisis. El estudio revela que la medicalización del control de fronteras oscurece la medición de la eficacia de las restricciones como herramienta de protección de la población, capaz de justificar el sacrificio de los derechos de los migrantes $\mathrm{VIH}$-positivos. Por otra parte, se observa que el aumento de la competencia de los profesionales de la salud contribuye a la disección terapéutica de la actividad médica, y se centra en las rutinas administrativas a expensas de los mejores intereses del migrante / paciente. Por último, observamos que los discursos y las prácticas médicas conocen y producen el migrante "sano", capaz de cruzar las fronteras estatales en el contexto de las relaciones de poder, que violan los derechos de los migrantes VIH-positivos.

Palabras-Ilave: Medicalización. Migración. Salud. Derechos humanos.

\section{Introdução}

A saúde tem sido um elemento comumente considerado na regulação dos fluxos migratórios internacionais. A entrada do estrangeiro no território dos países mostra-se condicionada, dentre outros requisitos, a seu estado de saúde, exigindo-se um controle de fronteiras apto a identificar migrantes "doentes" e "saudáveis", numa profunda articulação entre a medicina e o poder político. Neste processo, não apenas se protege - ou se tentar proteger - as comunidades domésticas contra doenças, alegadamente trazidas pelo estrangeiro; direitos humanos tornam-se passíveis de aviltamento e as práticas médicas se transformam, incorporando elementos que ultrapassam o exercício da cura.

Diante disso, este trabalho tem como objetivo analisar os impactos da medicalização no controle de fronteiras dos países, identificando possíveis implicações para a prática médica e para os direitos dos migrantes internacionais. Para tanto, assume como objeto de 
análise as restrições de viagem relacionadas ao HIV/AIDS, existentes na legislação imigratória do Canadá.

Além da introdução e considerações finais, o estudo se desenvolve em três seções. Na primeira delas, procede-se à análise das dimensões da medicalização, apresentandose as principais abordagens contemporâneas sobre o tema, que se integram na compreensão do fenômeno. Nessa esteira, discutem-se os aspectos relacionados à abordagem conceitual, à expansão da jurisdição social dos profissionais da saúde sobre uma parcela crescente da vida humana, e o emprego de discursos e práticas, não apenas para conhecer, mas também para produzir o corpo, num campo complexo de relações de poder.

Na segunda seção do trabalho, analisam-se as restrições de viagem relacionadas ao vírus HIV, existentes na legislação do Canadá. Descrevem-se as principais diretrizes relacionadas ao regramento da entrada e permanência de migrantes soropositivos no território do país, enfocando o desenvolvimento e consequências do exame médico imigratório. Neste ponto, demonstra-se que, embora a infecção por HIV não conste como causa explícita de exclusão sumária de estrangeiros, prevalece, como regra, a rejeição dos migrantes que vivem com o vírus.

Em sua terceira seção, o estudo argumenta que a medicalização do controle de fronteiras obscurece a aferição da eficácia das restrições, enquanto instrumento de proteção da população, capaz de justificar o sacrifício de direitos dos migrantes soropositivos. Além disso, observa-se que o aumento da jurisdição dos profissionais da saúde contribui para o esvaziamento terapêutico da atividade médica, centrada em rotinas administrativas em detrimento do melhor interesse do migrante/paciente. Finalmente, notase que discursos e práticas médicas conhecem e produzem o migrante "saudável", apto a cruzar as fronteiras do Estado, num contexto de relações de poder, que violam direitos dos migrantes soropositivos.

No que tange à metodologia utilizada, o estudo classifica-se como bibliográfico e documental. Quanto aos procedimentos metodológicos empregados, têm-se a leitura e a análise de materiais publicados sobre o tema, tais como livros, publicações em periódicos e artigos científicos, além do estudo e interpretação de informações que se encontram em documentos sem conteúdo analítico (leis, relatórios com dados estatísticos, etc.), conferindo-Ihes importância como fonte de análise. 
Com o estudo dos impactos da medicalização sobre o controle de fronteiras, este trabalho busca oferecer uma leitura crítica das práticas médicas no governo da mobilidade humana e suas implicações para a defesa (ou violação) dos direitos humanos do estrangeiro, iluminando aspectos obscurecidos pela forte retórica de proteção da saúde.

\section{Dimensões da medicalização: abordagens contemporâneas}

A medicalização pode ser analisada a partir do estudo de três abordagens distintas, mas interrelacionadas (01). A primeira enfatiza o elemento conceitual da medicalização; a segunda debruça-se sobre a expansão da jurisdição dos profissionais da saúde, especialmente, dos médicos; a última perspectiva, por sua vez, sem desconsiderar as abordagens anteriores, enfoca discursos e práticas médicas que conhecem e produzem o corpo. Como anteriormente dito, tais perspectivas não se excluem, enfatizando diferentes dimensões e efeitos do ato de medicalizar.

Enfocando o aspecto conceitual, Peter Conrad (02) explica que "Medicalization consists of defining a problem in medical terms, using medical language to describe a problem, adopting a medical framework to understand a problem, or using a medical intervention to treat it." Em outros termos, a medicalização descreve um processo através do qual situações passam a ser conceituadas, compreendidas e tratadas como problemas médicos, realizando-se intervenções médicas para sua resolução.

Além de definir e tratar problemas em termos de saúde e doença, a medicalização também promove a expansão da jurisdição social dos profissionais da medicina, aumentando sua influência no processo de tomadas de decisão sobre uma crescente parcela da vida humana (03):

The power vested in doctors is enormous: they make decisions about employ ability, fitness to marry and to have children, the right to have an abortion, the time a person is allowed to die, competence to enter into contracts, adopt children or rear one's own children, or about incarceration in mental asylums. Their authoritarian judgement is sought on correct eating, sexual behaviour and the use of leisure time. (04)

A influência dos médicos sobre uma gama cada vez maior dos fenômenos individuais e da população qualifica a medicina como uma importante instância de controle social, que constrói julgamentos objetivos, moralmente neutros, proferidos em nome da saúde (05). Reconhecendo-se que o controle social é problema relevante para o poder político, 
entende-se por que o Estado passa a estabelecer uma íntima relação com os profissionais da saúde, utilizando sua expertise para objetivos políticos e econômicos (06). estreitamento dos laços entre os profissionais da saúde e o Estado, faz com que a medicina assuma um lugar cada vez mais importante nas estruturas administrativas e nos mecanismos do poder, penetrando progressivamente em diferentes instâncias de seu exercício e demandando dos médicos cada vez mais a realização de atividades e tarefas administrativas fixadas pelo Estado (07).

A medicalização também pode ser analisada a partir dos discursos e práticas médicas através dos quais o corpo é conhecido e produzido, num reflexo de complexas relações de poder que se imbricam na construção do conhecimento. Ecoando o pensamento de Michel Foucault, esta perspectiva não compreende o poder apenas de maneira coercitiva e negativa. As relações de poder são também produtoras da realidade, da "verdade" e dos sujeitos. Além disso, estas relações não são externas aos processos econômicos, culturais, institucionais ou cognitivos. Efetivamente, poder e saber são mutuamente constituídos, de forma que não existem relações de poder sem suas correspondentes formas de conhecimento. O poder produz conhecimento e 0 conhecimento, por sua vez, produz o poder (08).

Este complexo de poder e conhecimento dá origem a modos particulares de entender o "eu", o corpo e a saúde, delineando a subjetividade e a identidade do indivíduo ao tempo em que desafia convicções sobre uma essência do sujeito, constituída independentemente de discursos e práticas (09). Na esteira deste entendimento é que se afirma que os discursos e práticas médicas não apenas conhecem o corpo, mas também o produzem, refletindo as relações de poder entre os elementos envolvidos. Medicalizar, portanto, é empregar discursos e práticas não apenas para conhecer, mas também para produzir o corpo num campo complexo de relações de poder.

\section{Restrições de viagem relacionadas ao HIV/AIDS: comentários sobre a lei imigratória canadense}

O Canadá é globalmente conhecido por ser um país rico, cujo sistema de saúde é um dos mais eficientes do mundo. A resposta canadense ao HIV também se destaca dentre as ações de saúde do Estado, tanto nacional quanto internacionalmente, destacando o sucesso do país em reduzir os casos de pessoas que vivem com o vírus dentro de suas 
fronteiras e sua posição de liderança na resposta global à AIDS (10). Em contradição com sua posição de destaque na resposta global ao HIV/AIDS, o Canadá, em 2002, modificou sua legislação imigratória para passar a exigir exame específico para deteç̧ão de HIV para estrangeiros que pretendem permanecer em seu território por mais de seis meses, dificultando o ingresso de migrantes soropositivos no país.

O exame médico imigratório é procedimento obrigatório para residência temporária (superior a seis meses) e permanente no Canadá. O procedimento é realizado, exclusivamente, por médicos credenciados, membros do Panel Physician Program, responsável por um detalhamento minucioso do estado de saúde do estrangeiro que intenta ingressar no território do país, através de testes que poderão incluir: exames físicos, mentais, revisão do histórico médico, exames laboratoriais e avaliação de registros de saúde apresentados pelo migrante.

Os resultados dos testes conduzem à elaboração do prontuário médico do migrante, que deverá ser encaminhado para o departamento de imigração canadense - Citizenship Immigration Canada (CIC). Recebido o prontuário, a equipe médica do referido departamento deverá proceder à avaliação das condições de saúde do estrangeiro, produzindo informações que informarão e legitimarão a tomada de decisão pela autoridade imigratória.

O objetivo do exame médico imigratório é verificar se a condição de saúde do migrante oferece perigo à saúde pública, perigo à segurança pública e/ou se implicará demandas excessivas por serviços sociais e de saúde. Sobre eventual excesso de demanda, a lei de imigração considera que o migrante será considerado inadmissível se a) os prováveis custos dos serviços sociais e de saúde que thes forem dispensados excederem aqueles do residente "médio" e b) sua demanda por estes serviços incrementar as listas de espera por serviços públicos, aumentando a mortalidade e morbidez no Canadá, pela demora ou impossibilidade de acesso a estes serviços pelos cidadãos canadenses e residentes permanentes. $O$ cálculo para aferir o excesso de demanda deve ainda, atender ao requisito temporal:

The estimated financial burden of someone applying to enter or remain in Canada is calculated over a five-year period from the date of the person's most recent immigration medical examination. According to the Regulations, the period of time may be extended to 10 years if there is evidence that 
significant costs are likely to be incurred beyond five years (such as in the case of HIV infection). The contributions the individual is expected to make to Canada, whether economic or social, are not taken into account. (11)

O Canadá não possui em sua legislação o HIV como causa explícita de exclusão sumária de migrantes. Além disso, pessoas que vivem com o vírus não são consideradas como um perigo à saúde ou segurança pública. A infecção pelo HIV, em regra, não impossibilita a entrada e permanência do estrangeiro no país por período igual ou inferior a seis meses, tendo em vista que, nestes casos, o estrangeiro não é elegível para uso de serviços de saúde; ocorre, assim, uma presunção de que não implicará demandas excessivas por estes serviços. Efetivamente, migrantes que desejam permanecer no país pelo referido período nem sequer submetem-se ao exame médico imigratório, reservado aos casos em que se verifique estado explícito de doença ou necessidade de hospitalização. A situação se modifica, no entanto, se o migrante intenta permanecer por mais de seis meses no Canadá ou fixar residência permanente no país.

Para permanência temporária superior a seis meses, o exame médico é obrigatório caso o migrante, no ano anterior ao pedido de entrada no Canadá, tenha residido - por seis ou mais meses consecutivos - num dos países identificados com maior prevalência de certas doenças comunicáveis. Se detectada infecção por HIV, o migrante se submeterá à avaliação da demanda excessiva e será declarado medicamente inadmissível se, durante a sua permanência, seja provável que ocasionará gastos públicos superiores à média dos cidadãos e residentes permanentes e/ou sua demanda por serviços públicos implique demora ou impossibilidade do acesso destes últimos à assistência médica e social, aumentando-se, assim, os índices de morbidade e mortalidade no Canadá. Nos casos de pedido de residência permanente, todo migrante deverá se submeter ao exame médico imigratório no qual se verificará, dentre outros, a existência de infecção por HIV/AIDS. Sendo o estrangeiro soropositivo, realizar-se-á a avaliação para determinar se sua entrada no país implicará demanda excessiva.

No entanto, a questão se problematiza, pois, segundo o Citizenship Immigration Canada $(\mathrm{CIC})$, em suas recomendações aos médicos responsáveis pelo exame de imigração,

HIV infection will frequently meet the CIC criteria of excessive demand due principally to the high cost of drug therapy. The costs of antiviral therapy are borne entirely by some provinces and territories and, in some provinces and 
territories, the patient pays a small proportion thereof. [...] It should be noted that a large majority of HIV-infected applicants will meet the criteria of excess demand, but there will be a small proportion which will qualify for admittance. Any applicant currently receiving ARV is inadmissible based on excess demand. (12)

Em outras palavras, migrantes que tenham iniciado tratamento com medicação antirretroviral serão inexcusáveis considerados medicamente inadmissíveis pelo critério da demanda excessiva. Aqueles que não o tenham começado passarão por uma avaliação que estimará se, no curso dos dez anos seguintes, iniciarão o referido tratamento. Caso afirmativo, também não deverão ser admitidos. Ademais, como os antirretrovirais são financiados pelo Estado - encontrando-se disponíveis para todos os cidadãos e residentes permanentes - a contratação de seguro médico ou plano de saúde não garante que o estrangeiro não fará uso dos serviços públicos, não servindo, assim, como prova para afastar o critério da demanda excessiva (13). Diante do exposto, compreende-se que as recomendações do $\mathrm{CIC}$ aos médicos responsáveis pelo exame imigratório estabelecem que - embora o HIV não conste como causa explícita de exclusão sumária de estrangeiros - a regra é que migrantes soropositivos sejam rejeitados e não que sua permanência seja permitida.

Deve-se registrar que - embora se submetam ao exame médico imigratório algumas classes específicas não se sujeitam ao critério da demanda excessiva por serviços médicos e de saúde. Refugiados e aqueles considerados em necessidade de proteção não serão avaliados quanto ao referido requisito; a seus cônjuges, companheiros e filhos dependentes também não se aplica o critério aludido. Igualmente, encontram-se isentos o cônjuge, companheiro ou filho dependente de cidadão canadense ou residente permanente, assim como os filhos por estes havidos.

\section{Medicalização do controle de fronteiras e restrições relacionadas ao HIV/AIDS: repercussões nas práticas médicas e nos direitos dos migrantes soropositivos}

Em países com barreiras, o ingresso de migrantes soropositivos é concebido como um problema que se explica a partir do discurso médico: estrangeiros com HIV são uma ameaça à saúde pública, causam demandas excessivas por serviços sociais e de saúde, além de aumentarem a morbidade e mortalidade de cidadãos e residentes, ao restringirem 
seu acesso a serviços médicos. Tomando a entrada de migrantes com o vírus como um "problema" a ser enfrentado, os Estados explicam as restrições de viagem a partir de uma terminologia médica, centrada nos conceitos de saúde e doença.

Mais não apenas isso: o problema também requer a atuação do aparato médico, incutido na estrutura do Estado, para que possa ser solucionado ou minimizado. Daí a contratação de médicos, credenciados para a realização do exame médico imigratório, cuja função fundamental é a detecção de doenças que inabilitem o migrante a cruzar as fronteiras nacionais. Ainda que as barreiras não possam "blindar" a população contra o HIV, detectar a infecção pelo vírus no estrangeiro e proibir sua entrada no país é medida que não pode ser dispensada.

O emprego do discurso médico para explicar as restrições relacionadas ao HIV e propor intervenções para sua administração - deve ser analisado com cautela, pois os rótulos "saúde" e "doença" detêm forte potencial de despolitizar os fenômenos humanos (14); neste sentido, afastam a problematização de premissas, que se tornam um fato dado e natural, promovendo acriticamente determinadas agendas que se valem da retórica da saúde. A despolitização trazida pelo emprego desta terminologia pode ser explicada pela potência do imperativo por uma vida saudável, livre de doenças, e pela força do apelo à saúde como bem necessário e desejável (15).

Medicalizar o controle de fronteiras enfraquece a problematização sobre se 0 estabelecimento de restrições de viagem é uma medida necessária e eficaz para a segurança da população, que justificaria 0 sacrifício de direitos dos migrantes soropositivos. Descrever o ingresso destes migrantes no território do país como um problema, inteligível a partir da referência à saúde da população, retira as barreiras de viagem do campo do questionamento, emprestando-lhe, a um só tempo, a força e a incontestabilidade do imperativo da saúde.

Consistindo noutro aspecto da medicalização, a expansão da jurisdição social dos profissionais da saúde, acompanhada da incorporação dos médicos nos aparatos estatais para exercício do poder, mostra-se nitidamente verificável no controle de fronteiras dos países com restrições de viagem relacionadas ao HIV/AIDS. Exemplo elucidativo é a realização do exame médico imigratório, no caso do Canadá, que incute os profissionais da saúde nas estruturas do Estado, realizando funções eminentemente administrativas, essenciais para o exercício do poder político na regulação dos fluxos migratórios: 


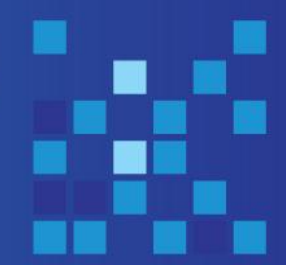

Their work is centrally organized around completing forms, collating documents, commissioning and attesting to the authenticity of diagnostic tests and safeguarding against identity fraud. Panel Physicians are first stage data collectors of information that enables informed decision-making about an applicant's medical inadmissibility to Canada. (16)

A realização de tarefas administrativas não se confunde com a perda de autoridade sobre a regulação das migrações. Ressaltando a importância dos médicos como atores centrais, na linha de frente do sistema de imigração, Laura Bisaillon e Carolyn Ells (17) explicam que

While the Panel Physicians' legal enforcement work is a great deal subtler than that of the border control agent (in the absence of a mandated uniform, weapon and physical position at a frontier), the two roles are organized within relations that embody the state's power. Through the work of both of these figures, important state gatekeeping functions are achieved.

A expansão da jurisdição social dos médicos, abrangendo o controle de fronteiras, fomenta inquietações. Embora muitas vezes a medicina se afirme como a ciência da cura, a serviço dos interesses dos pacientes, médicos nos serviços de saúde pública, ocupando cargos na maquinaria estatal, possuem, pela natureza do seu vínculo, diferentes fidelidades (18). No contexto das restrições de viagem relacionadas ao HIV, a lógica organizacional do trabalho é a detecção do vírus para fundamentar a exclusão do migrante soropositivo, mediante um esvaziamento da função terapêutica associada à atividade médica:

We see that the IME [immigration medical examination] has above all to do with medico-administrative duties to the state rather than therapeutic relations of care or the responsibility of patient best interest, and that these arrangements lead DMPs [designated medical practitioner] to work in contravention of principles guiding medical practice. (19)

Problemas como a ausência de consentimento informado, a falta de aconselhamento anterior e após os exames e a utilização dos dados médicos do migrante para fins diversos da análise clínica são procedimentos comumente observados no controle de fronteiras do Canadá (20). Os padrões médicos não são dispensados pelas leis, regulamentações e códigos de ética em vigência no país, mas se observa que tais disposições entram em conflito com evidências empíricas identificadas (21). 
No controle de fronteiras medicalizado, a incorporação dos médicos, no aparato estatal, ocorre mediante uma crescente realização de tarefas administrativas, através das quais o poder político é exercido, produzindo relações de empoderamento recíprocas. Este processo, no entanto, enfraquece a função terapêutica da clínica médica, vinculando a atividade dos profissionais da saúde aos interesses do Estado. O migrante soropositivo menos como sujeito de direitos e mais como corpo a ser governado - personifica o esvaziamento terapêutico da medicina no controle de fronteiras.

O exame médico imigratório é realizado através de um longo processo administrativo, centrado no preenchimento de formulários, juntada de documentos, interpretação de exames, elaboração de diagnósticos, verificação da autenticidade dos resultados, preparação de prontuários, além de outros procedimentos que o médico encarregado julgue necessário para construir o mais perfeito quadro da saúde do migrante. A partir deste emaranhado de dados, informações e interpretações é que se obtém - ou, em outros termos, se produz - o estrangeiro enquanto corpo "doente" ou "saudável" e, assim, aceitável ou inaceitável para cruzar as fronteiras do país. Como explica Laura Bisaillon (22) sobre o exame médico imigratório no Canadá:

A medical file can thus be seen as more than a report of disease status: it brings into view specific medical conditions, while excluding others, and creates the conceptual boundaries that define an acceptable immigrant.

Esta produção de conhecimento acerca do corpo do migrante não ocorre de maneira neutra sob o ponto de vista do poder. A realização do exame médico imigratório - e o desenvolvimento de todo o processo burocrático de coleta e interpretação de dados - toma lugar em meio a complexas relações entre o paciente/migrante e o médico/guardião das fronteiras do Estado. A racionalidade da interação entre o migrante e o médico é baseada na responsabilidade deste último em detectar doenças no corpo do estrangeiro, reportar resultados à autoridade imigratória para, finalmente, tomar lugar sua exclusão. Mediante uma sequência de práticas administrativas, que esvaziam o caráter terapêutico da clínica, o médico se faz autoridade, determinando condutas e impondo significados ao corpo do migrante.

O exercício do poder no exame médico imigratório é notório em momentos diversos da avaliação. Por exemplo, no caso do Canadá, o teste de HIV deve ser realizado 
mediante o consentimento informado do migrante, que deve conceder permissão para ser examinado quanto à infecção pelo vírus. No entanto, candidatos à imigração (ou permanência superior a seis meses) não têm escolha a não ser submeter-se ao procedimento, fazendo com que a solicitação do exame seja muito mais uma notificação prévia acerca da realização mandatória do teste do que uma requisição de consentimento (23).

O aconselhamento após a realização do teste de HIV, especialmente nos casos de detecção de infecção pelo vírus, é registrado em documento padronizado que atesta que o migrante foi orientado acerca de sua condição, incluindo estratégias de redução de riscos e uma discussão sobre a adoção de providências subsequentes. No entanto, como explica Laura Bisaillon e Carolyn Ells (24)

In signing this form, applicants attest to receiving HIV post-test counselling after diagnosis whether they have or not. Persons report feeling bound to sign the "Acknowledgement of post-testing counseling" form as part of their efforts to immigrate. [...] Indeed, applicants have little choice in the matter because a finalized medical file for an HIV-positive applicant must include this form signed by the applicant, the Panel Physician, and an expert witness or counsellor.

Conhecimento e poder se interrelacionam na complexidade do exame médico imigratório enquanto um conjunto de discursos e práticas médico-administrativas, direcionadas para conhecer e produzir o corpo do migrante como "saudável" ou "doente", "aceitável" ou "inaceitável" para cruzar as fronteiras dos países. Este entendimento e produção do corpo do estrangeiro toma lugar mediante relações de poder entre pacientes/migrantes e médicos/guardiões das fronteiras nacionais, indissociáveis da produção de conhecimento sobre o sujeito. Neste contexto, as restrições de viagem relacionadas ao HIV não apenas situam o corpo do migrante soropositivo nas fronteiras do Estado; efetivamente, o corpo carrega, em si próprio, a fronteira ao ser investido de marcadores biológicos que determinam sua inclusão ou exclusão do território dos países (25). 


\section{Considerações finais}

A análise dos impactos da medicalização sobre o controle de fronteiras permite identificar a transformação das práticas médicas, enquanto exercício da cura, incorporando elementos dissidentes de sua função terapêutica, numa profunda articulação entre os profissionais da saúde e o poder político. Além disso, com o estudo da medicalização do controle de fronteiras, verifica-se que os direitos humanos dos migrantes, que buscam sua entrada no território estrangeiro, são comumente negligenciados, num processo que obscurece $\mathrm{O}$ aviltamento de direitos, invocando a defesa da saúde nas comunidades domésticas. A análise das restrições de viagem relacionadas ao HIV, existentes na legislação canadense, permite aprofundar a compreensão do fenômeno.

Medicalizar é conceituar, compreender e tratar situações como problemas médicos, realizando-se intervenções médicas para sua resolução. A legislação imigratória canadense configura um controle de fronteiras medicalizado, pois a entrada de migrantes soropositivos é concebida enquanto problema que se explica por referência ao binômio saúde/doença, sendo abordado mediante a atuação do aparato médico, incutido na estrutura do Estado, responsável pela detecção do vírus no migrante que pretende cruzar as fronteiras nacionais.

No entanto, deve-se lembrar que descrever o ingresso de migrantes soropositivos no território dos países como um problema, inteligível a partir da referência à saúde da população, retira as barreiras de viagem do campo do questionamento, emprestando-Ihe, a um só tempo, a força e a incontestabilidade do imperativo da saúde. Neste contexto, temse que medicalizar o controle de fronteiras enfraquece a problematização sobre se as restrições de viagem são uma medida necessária e eficaz para a segurança da população, despolitizando seu estabelecimento; além disso, tal despolitização permite a promoção acrítica de agendas específicas, que se valem da retórica da saúde como fundamento de legitimidade.

Medicalizar o controle de fronteiras também significa estender a jurisdição dos profissionais da saúde para a tomada de decisões acerca do ingresso no território dos países. Neste processo, a medicina se fortalece enquanto instância de controle social e garante ao Estado a força de julgamentos objetivos, moralmente neutros, proferidos em nome da saúde. Numa relação de empoderamento recíproco, os médicos se infiltram 
progressivamente nas instâncias do poder, realizando, cada vez mais, atividades administrativas fixadas pelo poder político.

No controle de fronteiras canadense, o trabalho dos profissionais de saúde responsáveis pelo exame médico imigratório é eminentemente administrativo, centralizado no preenchimento de formulários, autenticação de resultados de testes e juntada de documentos necessários para a construção de um perfil médico do migrante. A realização de tarefas administrativas não se confunde, no entanto, com a perda da autoridade sobre a regulação das migrações. Os profissionais da medicina exercem grande influência na elaboração dos critérios de imigração, realizam os exames imigratórios, além de elaborarem pareceres sobre a possibilidade de demandas excessivas, que embasarão a decisão última tomada pelas autoridades imigratórias.

A expansão da jurisdição social dos médicos, abrangendo o controle de fronteiras, fomenta inquietações. Embora muitas vezes a medicina se afirme como a ciência da cura, a serviço dos interesses dos pacientes, médicos nos serviços de saúde pública, ocupando cargos na maquinaria estatal, podem possuir, pela natureza do seu vínculo, diferentes fidelidades. No contexto das restrições de viagem relacionadas ao HIV, a lógica organizacional do trabalho é a detecção do vírus para fundamentar a exclusão do migrante soropositivo, mediante um esvaziamento da função terapêutica associada à atividade médica. O migrante soropositivo - menos como sujeito de direitos e mais como corpo a ser governado - personifica o esvaziamento terapêutico da medicina no controle de fronteiras.

Por fim, pode-se dizer que medicalizar é empregar discursos e práticas não apenas para conhecer, mas também para produzir o corpo num campo complexo de relações de poder. A análise do funcionamento das restrições de viagem relacionadas ao HIV permite compreender a operação desta dinâmica. O exame médico imigratório é realizado através de um longo processo administrativo, centrado no preenchimento de formulários, juntada de documentos, interpretação de exames, elaboração de diagnósticos, verificação da autenticidade dos resultados, preparação de prontuários, além de outros procedimentos que o médico encarregado julgue necessário para construir o mais perfeito quadro da saúde do migrante. A partir deste emaranhado de dados, informações e interpretações é que se obtém - ou, em outros termos, se produz - o estrangeiro enquanto corpo "doente" ou "saudável" e, assim, aceitável ou inaceitável para cruzar as fronteiras do país. 
Esta produção de conhecimento acerca do corpo do migrante não ocorre de maneira neutra sob o ponto de vista do poder. A realização do exame médico imigratório - e o desenvolvimento de todo o processo burocrático de coleta e interpretação de dados - toma lugar em meio a complexas relações entre o paciente/migrante e o médico/guardião das fronteiras do Estado. A racionalidade da interação entre o migrante e o médico é baseada na responsabilidade deste último em detectar doenças no corpo do estrangeiro, reportar resultados à autoridade imigratória para, finalmente, tomar lugar sua exclusão. Mediante uma sequência de práticas administrativas, que esvaziam o caráter terapêutico da clínica, o médico se faz autoridade, determinando condutas e impondo significados ao corpo do migrante.

Direitos humanos são relativizados na execução do exame médico imigratório, tal como se verifica na manifestação de consentimento para sua realização, que mais se assemelha ao atendimento de uma notificação mandatória, tendo em vista que a recusa do migrante implicará sua inescapável rejeição. A ausência de aconselhamento anterior e posterior aos testes para detecção do HIV também ilustra como o aviltamento dos direitos dos migrantes toma lugar num controle de fronteiras medicalizado, que delimita os contornos de um migrante "saudável" e, por isso, aceitável para o ingresso na comunidade doméstica.

\section{Referências}

1. ELBE S. Security and Global Health. Cambridge: Polity; 2010.

2. CONRAD, P. Medicalization and Social Control. Annual Review of Sociology, 1992, 18 (1): 209-232.

3. ELBE, S. AIDS, Security, Biopolitics. International Relations, Londres, 2005, 19, (4): 403419.

4. SKRABANEK P. The Death of Humane Medicine and the Rise of Coercive Healthism. Suffolk: Social Affairs Unit; 1994.

5. ZOLA, I K. Medicine as an Institution of Social Control. The Sociological Review, 1972, 20, (4): 487-504.

6. SKRABANEK P. The Death of Humane Medicine and the Rise of Coercive Healthism. Suffolk: Social Affairs Unit; 1994. 
7. Foucault M. Microfísica do Poder. Tradução de Roberto Machado. 13. ed. Rio de Janeiro: Graal; 1998.

8. EDKINS J. Poststructuralism and International Relations: Bringing the Political Back In. Colorado: Lynne Rienner Publishers; 1999.

9. Elbe S. Security and Global Health. Cambridge: Polity; 2010.

10. Public Health Agency of Canada. HIV/AIDS. Canada, 2016. [Acesso em 08 maio 2015] Disponível em: http://www.phac-aspc.gc.ca/aids-sida/index-eng.php.

11. Canadian HIV/AIDS Legal Network. Canada's Immigration Policy and How it Affects People Living with HIV. Canadá, 2016. [Acesso em 12 dez 2015]

Disponível em: http://www.aidslaw.ca/site/canadas-immigration-policy-as-it-affects-peopleliving-with-hiv-questions-and-answers/.

12. Canadá. Citizenship Immigration Canada. Canadá, 2016. [Acesso em 02 dez.2014] Disponível em: http://www.cic.gc.ca/english/.

13. Canadian HIV/AIDS Legal Network. Canada's Immigration Policy and How it Affects People Living with HIV. Canadá, 2016. [Acesso em 12 dez 2015]

Disponível em: http://www.aidslaw.ca/site/canadas-immigration-policy-as-it-affects-peopleliving-with-hiv-questions-and-answers/.

14. ZOLA, I K. Medicine as an Institution of Social Control. The Sociological Review, 1972, 20, (4): 487-504.

15. HOWEL, A. Toward an International Political Sociology of Health and Medicine. International Political Sociology, 2012, 06, (3): 315-316.

16. BISAILLON, L. ELLS, C. When Doctoring is not about Doctoring: An Ethical Analysis of Practices Associated with Canadian Immigration HIV Testing. Public Health Ethics, 2014, 11, (1): 01-11.

17. BISAILLON, L. ELLS, C. When Doctoring is not about Doctoring: An Ethical Analysis of Practices Associated with Canadian Immigration HIV Testing. Public Health Ethics, 2014, 11, (1): 01-11.

18. SKRABANEK P. The Death of Humane Medicine and the Rise of Coercive Healthism. Suffolk: Social Affairs Unit; 1994.

19. BISAILLON, L. Contradictions and Dilemmas Within the Practice of Immigration Medicine. Canadian Journal of Public Health, 2013, 104, (1): 45-51. 
20. BISAILLON, L. ELLS, C. When Doctoring is not about Doctoring: An Ethical Analysis of Practices Associated with Canadian Immigration HIV Testing. Public Health Ethics, 2014, 11, (1): 01-11.

21. BISAILLON, L. Contradictions and Dilemmas Within the Practice of Immigration Medicine. Canadian Journal of Public Health, 2013, 104, (1): 45-51.

22. BISAILLON, L. Contradictions and Dilemmas Within the Practice of Immigration Medicine. Canadian Journal of Public Health, 2013, 104, (1): 45-51.

23. BISAILLON, L. ELLS, C. When Doctoring is not about Doctoring: An Ethical Analysis of Practices Associated with Canadian Immigration HIV Testing. Public Health Ethics, 2014, 11, (1): 01-11.

24. BISAILLON, L. ELLS, C. When Doctoring is not about Doctoring: An Ethical Analysis of Practices Associated with Canadian Immigration HIV Testing. Public Health Ethics, 2014, 11, (1): 01-11.

25. Vieira M. Biopolíticas de Exclusão: HIV/AIDS no controle de fronteiras [Dissertação]. Salvador: Programa de Pós-graduação em Relações Internacionais, Universidade Federal da Bahia; 2016.

Como citar este artigo:

Vieira MM. Medicalização e migrações internacionais: proteção de direitos e práticas de saúde no controle de fronteiras. Revista Cadernos Ibero-Americanos de Direito Sanitário. 2016 dez., 5 supl. 1:165-181. 